\title{
Decision Trees for Use in Childhood Mental Disorders
}

Henry A. Doenlen, MD

Thomas Jefferson University Hospital

Follow this and additional works at: https://jdc.jefferson.edu/jeffjpsychiatry

Part of the Psychiatry Commons

Let us know how access to this document benefits you

\section{Recommended Citation}

Doenlen, MD, Henry A. (1986) "Decision Trees for Use in Childhood Mental Disorders," Jefferson Journal of Psychiatry. Vol. 4 : Iss. 1 , Article 9.

DOI: https://doi.org/10.29046/JJP.004.1.006

Available at: https://jdc.jefferson.edu/jeffjpsychiatry/vol4/iss $1 / 9$

This Article is brought to you for free and open access by the Jefferson Digital Commons. The Jefferson Digital Commons is a service of Thomas Jefferson University's Center for Teaching and Learning (CTL). The Commons is a showcase for Jefferson books and journals, peer-reviewed scholarly publications, unique historical collections from the University archives, and teaching tools. The Jefferson Digital Commons allows researchers and interested readers anywhere in the world to learn about and keep up to date with Jefferson scholarship. This article has been accepted for inclusion in Jefferson Journal of Psychiatry by an authorized administrator of the Jefferson Digital Commons. For more information, please contact: JeffersonDigitalCommons@jefferson.edu. 


\title{
Decision Trees for Use in Childhood Mental Disorders
}

\author{
Henry A. Doenlen, M.D.
}

The third edition of The Diagnostic and Statistical Manual of Mental Disorders (DSM-III) (1) provides specific diagnostic categories for use in childhood mental disorders, even though these diagnoses are not limited to children. In addition, many of the diagnostic categories used for adults are considered appropriate for use in children. DSM-III instructs the clinician to diagnose children by first considering the section "Disorders First Evident in Infancy, Childhood, or Adolescence" before considering the disorders described elsewhere. However, this may lead to problems because some major diagnostic categories such as affective disorders and schizophrenia are not included in the childhood section. This may lead some clinicians to overlook a more accurate diagnosis outside the childhood section, i.e., using Overanxious Disorder instead of Major Depression.

In an attempt to help the clinician to understand the structure of the classification system, DSM-III contains a set of decision trees. Although these trees may be useful for adult diagnosis, they are not quite as useful in diagnosing children. The main problem is that children generally are brought to psychiatrists with behavioral complaints which are related by their parents. Children are more likely than adults to act out their feelings in non-specific ways. For example, a child's verbalization of worries to his parents may be a symptom of Separation Disorder, Major Depression, or Overanxious Disorder. Use of the decision trees in DSM-III would require the clinician to make an initial distinction between anxious mood and depressed mood. This is difficult with children, who often are unable to verbally label their feelings. Another factor complicating diagnosis in children is their greater imagination leading to the assessment of hallucinations or delusions which may not necessarily indicate psychosis.

This article proposes an alternate set of decision trees that may be helpful in the diagnosis of mental disorders in children and adolescents under the age of 18 years. Like the DSM-III decision trees, these trees are only approximations of the actual diagnostic criteria. Thus, they are not meant to replace the actual diagnostic criteria in DSM-III.

The following decision trees should be used in the order presented. Thus,

Dr. Doenlen wrote this paper while a senior child fellow at Thomas Jefferson University, Philadelphia, Pennsylvania. 
the first tree would apply to any emotional or behavioral problem, the next to psychotic problems, then speech and language problems, and so on. Generally, use of an individual tree should result in only one diagnosis from that tree. Exceptions to this are tree branches labeled "continue," which indicates that the remainder of the tree should be examined even if a diagnosis was already indicated. All decision trees should be used in the diagnostic considerations regarding each patient because a patient may have more than one applicable diagnosis. Each diagnosis suggested by the trees should be confirmed by application of the actual DSM-III diagnostic criteria. Special care should be taken to apply the exclusionary criteria for age or other features as stated in DSM-III. The tree for Organic Brain Syndromes would be the same as published in DSM-III, so it will not be repeated here. Also, the possibility of psychosexual disorders should be remembered even though there is no tree for them here or in DSM-III.

The author's hope is that these decision trees may be useful for residents studying child psychiatry. In addition, this article should stimulate discussion among clinicians who have different ideas about child psychiatric diagnosis. Such ideas may be used to modify and improve this attempt to organize the diagnostic categories. If individual residents find the trees helpful, a study might eventually be done comparing diagnosis made with and without the decision trees. The diagnosis may be compared for accuracy with those obtained by the child psychiatry faculty. In the field trials for DSM-III, the overall kappa coefficients of agreement for phase one was .68 and phase two was .52. Spitzer considered kappa values of 0.7 and above to indicate "good agreement." Perhaps the use of either the decision trees presented here or a series of modified decision trees may improve the diagnostic agreement among clinicians.

\section{CHILD MENTAL DISORDER DECISION TREES}

\section{1) Diagnostic Considerations for All Symptoms}

Any behavioral or emotional symptom?

+yes

Symptoms under voluntary control?

+ no +yes

$+\quad$ Goal only to take patient

+ role?

$+\quad+$ no

$+\quad$ Goal obviously recognizable?

$+\quad+$ no

Known organic etiology (including neurologic and other physical disease as

well as drug or alcohol ingestion?)---_-_-yes: consider organic brain synd. + no consider other categories 
2) Psychosis Decision Tree

Delusions, hallucinations, loose associations, or incoherence?

+ yes

School, social, and self-care

functioning deterioration?

+ no +yes

$+\quad$ Delusions, hallucinations,

$+\quad$ loose associations, or

$+\quad$ incoherence?

$+\quad$ Duration 6 mo.? -

$+\quad+$ no

$+\quad$ Duration 2 wks.?

yes: Schizophreniform disorder

$+\quad+$ no

$+\quad$ After significant stress?

$+\quad+$ no

Initially manic or depressed? -yes: Brief reactive psychosis

$$
+ \text { no }
$$

Paranoid delusions without hallucinations?--yes: Paranoid disorder + no

School, social, and self-care functioning

deterioration?

+ no

consider Anxiety, other categories

\section{3) Language Decision Tree}

Speech or language difficulties?

+ yes

Delusions, hallucinations, loose

associations?

$$
+ \text { no }
$$

Onset before age 2.5 yrs.?

$$
\text { +no+yes }
$$

$+\quad$ Pervasive lack of

$+\quad$ responsiveness to people? ----------yes: Infantile autism

$+$

Gross impairment of sustained social

relationship before age 12 yrs.? -yes: Childhood onset pervasive development dis.

$$
\text { + no }
$$

Multiple distortions of development

involving language and social functioning? --yes: Atypical pervasive dev. dis. + no

Magical thinking, inadequate rapport, odd

speech, suspiciousness?-----o---o--os: Schizotypal personality

$$
+ \text { no }
$$


Ability to comprehend spoken language, and to speak?

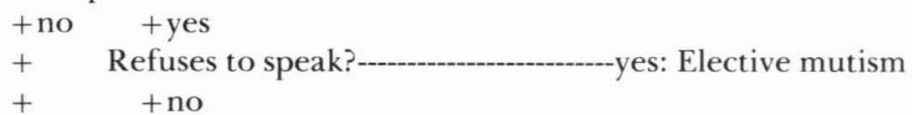

$+\quad+$ no

$+\quad$ Disruption of rhythmic flow of

$+\quad$ speech?

$+$

IQ less than 70?

+ no

Hearing impaired?

+ no

Failure to develop consistent articulation

with intact comprehension and expression? -yes: Develop. articulation dis.

+ no

Intact comprehension with failure of vocal

expression?

--yes: Expressive develop. lang. dis.

+ no

Failure of comprehension and expression? --yes: Receptive develop. lang. dis.

4) Relationship Problem Decision Tree

Impaired or problematic relationships?

+ yes

Delusions, hallucinations, loose associations,

incoherence?

+ no

Age less than 8 mo., apathetic after neglect,

reverses with caretaking? ----ones: Reactive attach. dis. infancy

+ no

Pervasive social impairment with odd

behavior, language, or speech deficits

before age 12 yrs.?

+ no +yes

$+\quad$ Onset before age 2.5 yrs.?----------yes: Infantile autism

$+\quad+----$ no: Pervasive developmental disorder

$+$

Speech or language problems with parents?-yes: consider language prob. tree + no

Depressed mood?

+ no

Magical thinking, illusions, odd speech,

suspiciousness?

+ no

-yes: consider Psychosis

Anxious with other people?

+ no + yes

$+\quad$ Age greater than 2.5 yrs? -----------no: assume normal stranger anxiety 


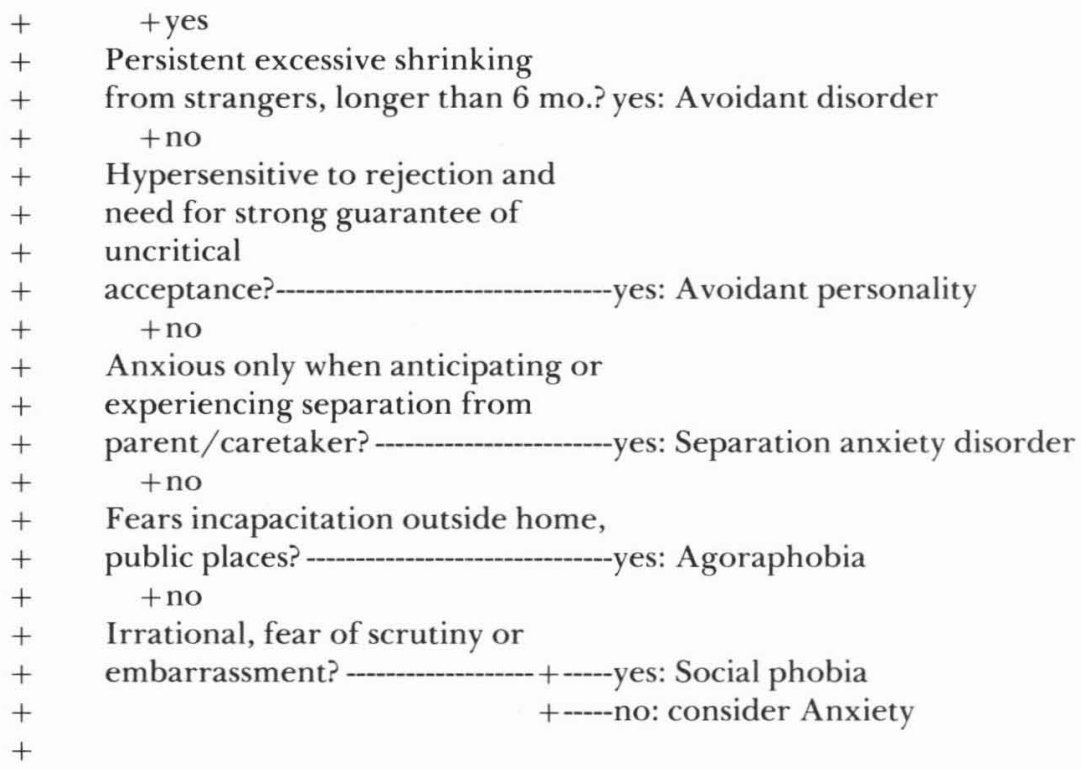

Occurred after significant stress less than

3 mo. ago?

yes: Adjustment reaction with withdrawal

+ no

Avoidance of peer relationships?

+ no

No mental disorder in patient?

+ no +yes

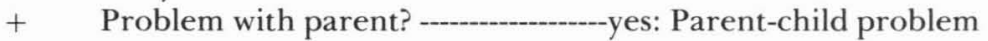

$+\quad+$ no

$+\quad$ Problem with spouse? ---arital problem

$+\quad+$ no

$+\quad$ Problem with other family

$+\quad$ member? ------yes: Other sp. family circumstances

$+\quad$ +---no: Other interpersonal problem

$+$

Long term relationship impairment?---------no: consider other categories +yes

Emotionally cold, aloof, close to less than

3 people? ---yes: Schizoid personality dis. + no

Unstable relationships, angry outbursts?

+ no +yes

$+\quad$ Impulsive, self-damaging? --------yes: Borderline personality dis.

$+\quad+$ no

$+\quad$ Grandiose self-importance,

$+\quad$ exploitative? - -

$+\quad+$ no

$+\quad$ Overly dramatic, demanding?------yes: Histrionic personality dis. 
Perfectionistic, excessive devotion to

productivity, rigid? yes: Compulsive personality dis.

+ no

Passively allows others to assume own

responsibility, lacks self-confidence?---------yes: Dependent personality dis.

+ no

+

5) Anxiety and Overactivity Decision Tree

Anxious or overactive?

+ yes

Delusions, hallucinations, or thought

disorder?

-yes: consider Psychosis

+ no

Anxiety in reaction to identifiable stressor

within $3 \mathrm{mo}$ ?----os: Adjust. dis. with anxious mood + no

Short attention span, impulsive, and

hyperactive?

-yes: Attention deficit disorder

+ no

Anxiety when anticipating or experiencing

separation from parents or caretaker? --------yes: Separation anxiety disorder + no

Persistent shrinking from strangers? ----------yes: Avoidant disorder

+ no

Persistent generalized anxiety? ----o---o----yes: Overanxious disorder

+ no

Fears incapacitation outside home or public

places?

+ no + yes

+ Panic attacks?--------------- +--yes: Agoraphobia with panic attacks

$+\quad$ +---no: Agoraphobia w/o panic attacks

$+$

Excessive fears of scrutiny or

embarrassment? ---os: Social phobia

+ no

Fear of single object or situation?

-yes: Simple phobia

+ no

Recurrent persistent ego-dystonic thoughts

or behaviors?

+ no

Reexperiencing a significant trauma with detachment and diminished outside

interests?

-yes: Post-traumatic stress syndrome

+ no

Anxiety about physical illness?

+ no -yes: consider physical illness tree 
Distress regarding identity issues? --_---o----yes: Identity disorder + no

Discrete panic attacks? ------o---on: Panic disorder + no

Overactive without anxiety? no: Consider other categories, including Generalized Anxiety Disorder + yes

More talkative, decreased sleep, overoptimism? no: consider other categories + yes

Grandiosity or flight of ideas yes: Manic psychosis no: Hypomanic

6) Depression and Hypoactivity Decision Tree

Depressed or hypoactive?

+yes

Dysphoric mood, appetite change, sleep

change, slowed thinking, loss of pleasure

for greater than 2 wks.?------yes: Major depression

+ no

$+\quad$ Hallucinations, delusions? ----- +----no: Major depression

$+\quad+----y e s:$ Maj. depression with psychotic features

$+$

Hallucinations, delusions? + no

Reexperiencing significant trauma,

detachment, hyperalert? ------os: Post traumatic stress dis.

+ no

Normal reaction to death of loved one?-------yes: Uncomplicated bereavement + no

Reaction to identifiable stressor within

3 mo.?yes: Adjust. dis. with depression + no

Periods of hypomania and depression for greater than 2 yrs.? + no $+$ yes: Cyclothymic disorder no: Atypical depression

\section{7) Learning Difficulties Decision Tree}

\section{Learning difficulties?}

$$
+ \text { yes }
$$

Neurologic exam reveals specific neurologic disorder? yes: Axis III neurologic diagnosis + continue

Delusions, hallucinations, loose associations, or incoherence? -yes: consider Psychosis

$$
+ \text { no }
$$


IQ less than 70 ?

+ no

Reading more than $1 \mathrm{yr}$. behind that

suggested by IQ? + no

Arithmetic more than $1 \mathrm{yr}$. behind that

suggested by IQ? + no

Short attention, impulsivity, hyperactivity?--yes: Attention deficit disorder with hyperactivity

+ no

Disobedient or aggressive?

+ no

Depression, anxiety, or social impairment? --yes: consider appropriate category + no

Learning problem in reaction to

identifiable stress within 3 mo.? ----------yes: Adjustment disorder with academic inhibitions

+ no

Distress about identity issues?

+ no

IQ between 71 and 84 ? --_-----yes: Borderline intelligence -yes: Identity disorder

+---no: Academic problem

\section{8) Disobedience Decision Tree}

Disobedience, impulsivity, or aggression?

+ yes

Delusions, hallucinations, loose associations,

or incoherence?

--yes: consider Psychosis

+ no

IQ less than 70? -yes: Mental retardation

+ no

Short attention, impulsivity, hyperactivity?--yes: Attention deficit disorder + no

Violation of rights of others outside of soci-

etal norms?

+ yes + no

$+\quad$ Impulsive gambling only? -----------yes: Pathological gambling

$+\quad+$ no

$+\quad$ Impulsivity in potentially

$+\quad$ self-damaging acts, unstable

$+\quad$ relationships, intense anger,

$+\quad$ affective instability?

$+\quad+$ no

$+\quad$ Disobedient, negativistic,

$+\quad$ provocative opposition to 
$+\quad$ authority? --_-_-_-_-_-_os: Oppositional disorder

$+\quad+$ no

$+\quad$ Procrastination, intentional

$+\quad$ inefficiency, stubbornness? ---_----yes: Passive-aggressive pers. dis.

$+$

Reaction to identifiable stressor within

3 mo.?

+ no + yes

$+\quad$ Includes disturbance of

$+\quad$ emotions? -------yes: Adj. dis. with mixed emotions and conduct

$+$

+----no: Adj. dis. with disturbance of conduct

Repetitive acts?

+no +yes

$+\quad$ Fire setting without gain; only?------yes: Pyromania

$+\quad+$ no

$+\quad$ Stealing without gain; only?--------yes: Kleptomania

$+\quad+$ no

$+\quad$ Persistent violations of rights of

$+\quad$ others? ---_---yes: Conduct disorder

$+$

Otherwise normal behavior with episodes of

loss of control of aggression resulting in serious assault or destruction?

+ no + yes

$+\quad$ More than 1 episode?--------- +---yes: Intermittent explosive dis.

$+\quad+----n o:$ Isolated explosive disorder

$+$

Isolated antisocial acts? -----------------yes: Antisocial behavior

9) Personality and Memory Change Decision Tree

Personality change or memory loss?

+yes

Delusions, hallucinations, loose associations,

or incoherence? -- -

+ no

More than 1 distinct personality, each

dominant at particular times? ---------------yes: Multiple personality + no

Sudden inability to recall the past; travel,

and new identity assumed? ---------------yes: Psychogenic fugue + no

Sudden inability to recall extensive personal

information?

+ no

Depersonalization episodes (feelings of 
unreality with impairment of functioning)?--yes: Depersonalization disorder + no

consider other categories, including depres-

sive and anxiety disorders

10) Eating Problem Decision Tree

Eating problem?

+ yes

Psychosis? -yes: consider Psychosis

+ no

Change in appetite and weight in the

presence of depression? + no

Repeated eating of nonnutritive substance? -yes: Pica + continue

Weight loss?

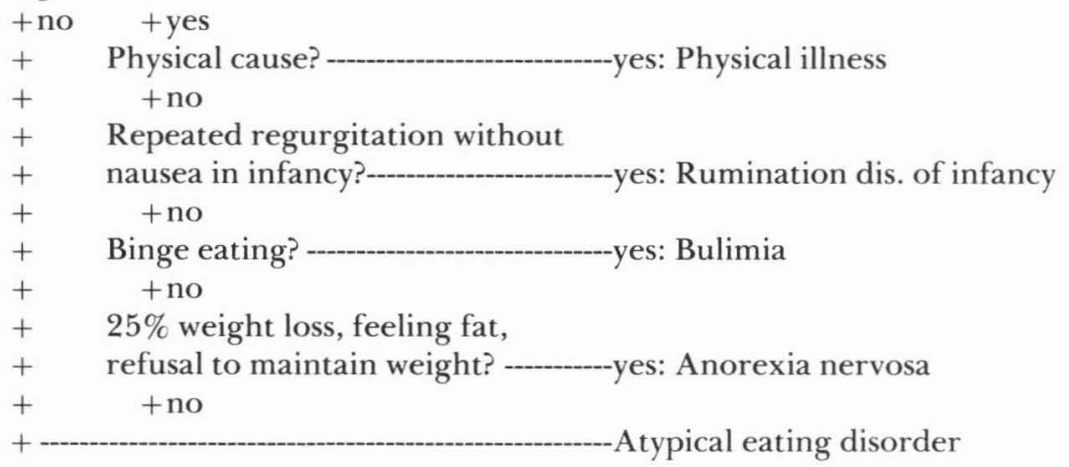

11) Sleep Problem Decision Tree

Sleep problems?

+ yes

Repeated problems 30-200 min. after sleep

onset?

+ no + yes

$+\quad$ Walking during sleep?

$+\quad+$ no

$+\quad$ Abrupt awakening with anxiety

$+\quad$ and autonomic arousal? ------------yes: Sleep terror

$+\quad+$ no

consider other categories, including depres-

sive and anxiety disorders

12) Physical Complaints Decision Tree

Irrational complaints of physical symptoms?

+ yes 
Symptom under voluntary control?

+ no +yes

$+\quad$ Is goal to assume patient role? ------no: Malingering

$+\quad+$ yes

$+\quad$ Multiple hospitalizations?------ +---yes: Chronic factitious phys. sx.

$+\quad$ +---no: Atypical factitious phys. sx.

$+$

Actual physical condition worsened by

psychologic factors?

-yes: Psychol. affect physical cond.

+ no

Severe prolonged pain not physical or in

excess of physical? ---os: Psychogenic pain

+ no

Alteration in physical functioning

suggesting physical disorder?------------------yes: Conversion disorder + no

Seeking medical attention for multiple

symptoms for several years?---_-_-_----yes: Somatization disorder + no

Fearful inappropriate belief of having

serious disease?-------yes: Hypochondriasis

+---no: Atypical somatization dis.

13) Other Physical Problems Decision Tree

Other physical problems?

+ yes

Repeated involuntary urine voiding?----------yes: Enuresis

+ continue

Repeated passage of feces into inappropriate

place?

+ continue

Recurrent repetitive, involuntary, rapid,

purposeless, movements? ----o-os: Tic disorders

+ continue

Repetitive voluntary movements?

yes: Atyp. stereotyped movement dis.

\section{REFERENCES}

1. American Psychiatric Association. Diagnostic and Statistical Manual of Mental Disorders. Third edition. 1980. 